\title{
Experiment with Axiom Propeller in Cavitation Tunnel
}

\author{
Kwang-Cheol Seo* \\ * Emerson Cavitation Tunnel, School of Marine Science and Technology, Newcastle University, UK
}

Abstract : The Axiom propeller is a unique 3 bladed propeller and it enables to generate the same amount of thrust going ahead as it does going astern because of its 's' type skew-symmetric blade section. A earlier variant of the design (Axiom I propeller) performed a low propeller efficiency, maximum $35 \%$ efficiency, and further blade outline design was carried out to achieve a higher efficiency. The optimized new blade outline (Axiom II propeller) has more conventional Kaplan geometry shape than Axiom I propeller. Model tests of open water performance and propeller cavitation for both propellers were conducted at Emerson Cavitation Tunnel in order to compare their performances. Experiment results revealed that Axiom II propeller provides a maximum $53 \%$ efficiency and provides better efficiency and cavitation performance over the Axiom I propeller under similar conditions.

Key Words : Skew symmetric blade section, Open water performance, Cavitation, Axiom propelle, Kaplan geometry

\section{Introduction}

The Axiom propeller is a unique 3 bladed propeller that is designed for the low speed/lightly loaded, narrow boat and sailboat markets. Since the propeller was first launched in 2008, around 700 vessels have now been fitted with an Axiom propeller, with inland vessel owners comprising a major chunk of Axiom's customer base. The design differs from the more conventional propeller geometry of aerofoil type propeller sections in-so-far as it has an unusual 's' type skew-symmetric blade section with spade like blades outlines shown in Fig. 1 This symmetry allows the propeller to generate the same amount of thrust going ahead as it does going astern, one of its key design features, enabling it to be a bi-directional thrust propeller. Fig. 2 shows an Axiom propeller design fitted to a narrow boat. A narrow boat is a boat of distinctive design, made to fit the narrow canals of Great Britain. The unique propeller performs well in low speed, sailing in restricted waters, all often at typical 6-9 knots.

Advocates for the Axiom design claim improved handling, greater fuel efficiency, reduced 'prop-walk' and impressive stopping performance for their vessels. Vessels such as barge and narrow boat operating in costal and canal require a good resistance performance at low speed, manoeuvrability and stopping which are the key performance requirements (Chun et al., 1997; Kim et al., 2001). And it is a common problem with conventional propellers which have difficulty in staying straight in reverse as well.

† skc0076@hotmail.com,0044-191-222-6726

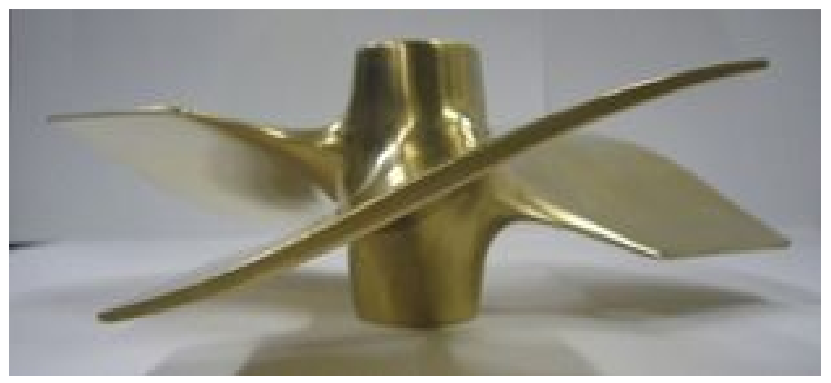

Fig. 1. The Axiom propeller showing the unusual 's' type section on a 3 blade propeller.

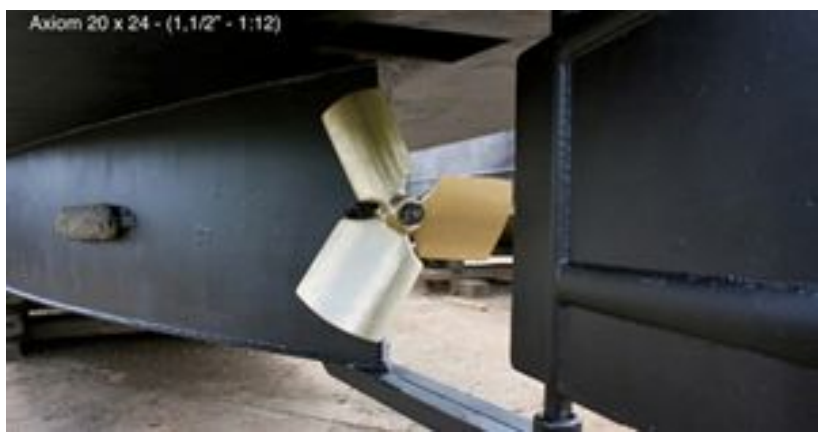

Fig. 2. Axiom propeller fitted to a narrow-boat.

The design for the Axiom propeller was developed somewhat heuristically and the parent design suffered from a low open water efficiency of around $35 \%$. This was encouraging given that no experimental testing or validation in a research facility had been performed adopting a trial and error style of optimisation; all of which encouraged the designers to pursue further optimisation. For the early Axiom design, trials were conducted in a simple manner as reported by Langley (2009). For these 
Experiment with Axiom Propeller in Cavitation Tunnel

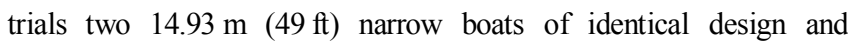
powering were used; the first fitted with a conventional propeller, the second with the Axiom propeller. The boats were run along the same stretch of canal approximately $100 \mathrm{~m}$ apart. The trials were conducted by running the boats at the same shaft rotational rate (rpm) and timed between various destinations. The canals were typically shallow, with bank and depth effects; the conditions of the hulls were not specified. A series of return runs were also performed in an effort to reduce the effects of current on the results. However despite this rudimentary trials procedure, the tests showed the benefit of the Axiom design. When compared to the conventional design the Axiom propeller showed an increase in speed and handling for the same rpm but with a drop in vibration. Fig. 3 shows the boats during the trial at the same rpm. The trials team noted the different wave patterns and wake created by the various designs, visible in Fig. 3, but failed to recognize that the wave patterns were speed dependent and would change from favourable to unfavourable depending upon Froude number, and several other factors.
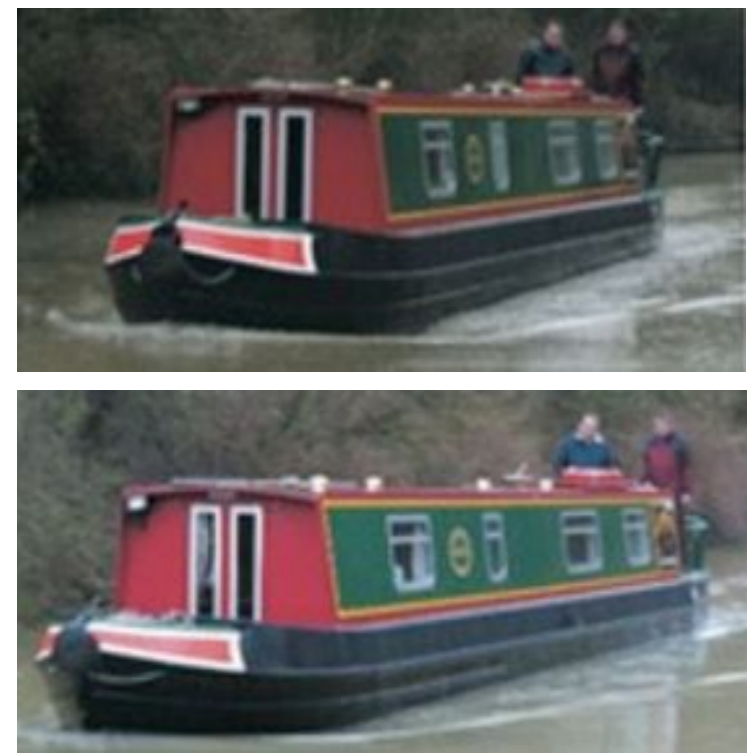

Fig. 3. Performance trials with the regular propeller (top) and the Axiom propeller (bottom) at the same rpm.

And the two test propellers were also very different in their stopping ability. The conventional propeller stopped the boat from walking pace in one ship length; with the Axiom it was half of that. Whilst the design is appealing for its manoeuvring characteristics, its cavitation and loading potential were unknown beyond these simplistic trials. To fully understand the capabilities and the limitations of the blade design a series of scientific trials and investigations were required. In collaboration with the Emerson Cavitation Tunnel, Axiom Propellers optimised their blade design to reduce the spade like blade outline shown in Fig. 4 to a more conventional Kaplan geometry shown in Fig. 5. The Axiom I propellers are suited to heavy, displacement hulls like canal boats whilst the Axiom II propellers are suited to faster hulls such as yacht and sailboats. Table 1 shows the full scale values of a boat suitable for the Axiom II propeller.

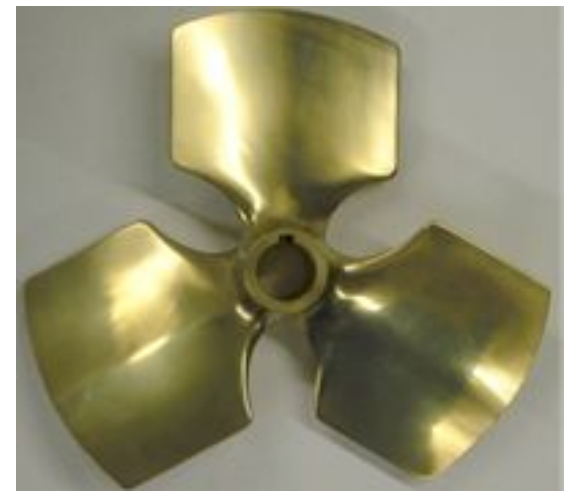

Fig. 4. The Axiom I propeller design.

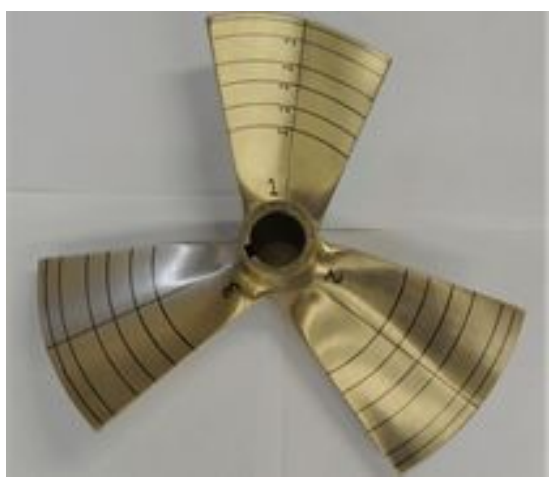

Fig. 5. The Axiom II propeller design.

Table 1. Full scale values of a boat suitable for the Axiom II propeller

\begin{tabular}{l|c}
\hline Length (waterline) & $11.73 \mathrm{~m}$ \\
\hline Breadth & $4.22 \mathrm{~m}$ \\
\hline Draught & $2.2 \mathrm{~m}$ \\
\hline Shaft submergence & $1.65 \mathrm{~m}$ \\
\hline Shaft inclination & $5^{\circ}$ \\
\hline Displacement & 22 Tonnes \\
\hline Stern type & Canoe \\
\hline Engine & $75 \mathrm{hp} @ 3800 \mathrm{rpm}$ \\
\hline Reduction gear & $2.63: 1$ \\
\hline Maximum propeller rpm & 1440 \\
\hline Speed over ground & $8.25 \mathrm{knots}$ \\
\hline
\end{tabular}




\section{Kwang-Cheol Seo}

It was hoped that the new design could be more efficient and operate at higher speeds whilst still utilizing the bi-directional thrust capability. To understand the limitations of this new design, a further series of model tests were performed to assess the improvements in performance due to these design changes. Following this introduction in Section 1, Section 2 gives the test set-up and Section 3 gives the results of the open water performance tests including the first two quadrant data for this propeller and a short review of the cavitation observations in the 1st and 2nd quadrant and Section 4 draws conclusions from the results.

\section{Implementation of experiment}

The propeller tests were conducted in the Emerson Cavitation Tunnel (ECT) in May 2012 as reported by Sampson et al. (2012). The ECT is a closed circuit depressurised tunnel located within the Newcastle University. A schematic of the tunnel is given in Fig. 6. The ECT has a measuring section of $3.2 \mathrm{~m} \times$ $1.2 \mathrm{~m} \times 0.8 \mathrm{~m}$; a contraction ratio of $4.274: 1$ and is therefore considered a medium sized facility. During recent years, numerous improvements to the instrumentation equipment and measuring section have taken place all increasing the capabilities of the facility. In 2008, the tunnel was upgraded with the installation of a new measuring section, guide vanes, honeycomb, quick degassing system and automated control system. The basic specifications for the tunnel are given in Table 2 and the details of this recent upgrading were reported in Atlar (2011).

The propeller tests were performed using a large H45 Kempf and Remmers open water dynamometer at atmospheric conditions. The propeller used in the experiment was a full-scale model of the Axiom propeller shown previously in Fig. 5, designated Axiom II. The model propeller was manufactured by Axiom

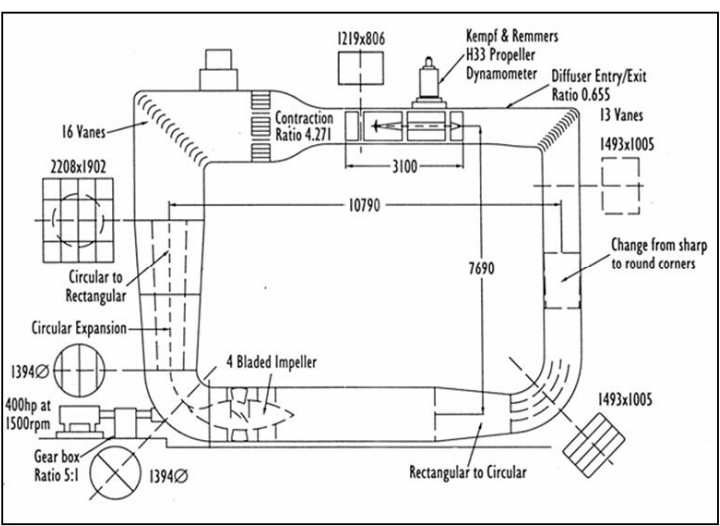

Fig. 6. Emersion Cavitation Tunnel schematic.
Propellers in bronze to fit a dynamometer shaft. The propeller was $300 \mathrm{~mm}$ diameter with a pitch to diameter ratio $(\mathrm{P} / \mathrm{D})$ of 1.0 and a blade area ratio (BAR) of 0.5 . Table 3 and 4 give the main particulars of the propeller whilst Fig. 7 shows the propeller of Axiom II prior to the test.

Table. 2 Emerson Cavitation Tunnel specification

\begin{tabular}{l|l}
\hline Tunnel & Emerson Cavitation Tunnel \\
\hline Facility Type & Vertical, closed Circulating \\
\hline Test section $(\mathrm{LxBxH})$ & $3.10 \mathrm{~m} \times 1.22 \mathrm{~m} \times 0.81 \mathrm{~m}$ \\
\hline Contraction ratio & 4.271 \\
\hline Drive system & 4 Bladed axial flow impeller \\
\hline Main pump power & $300 \mathrm{~kW}$ \\
\hline Impeller diameter & $1.4 \mathrm{~m}$ \\
\hline Maximum velocity & $8 \mathrm{~m} / \mathrm{s}(15.5 \mathrm{knots})$ \\
\hline Abs. pressure range & $7.6 \mathrm{kN} / \mathrm{m}^{2}$ to $106 \mathrm{kN} / \mathrm{m}^{2}$ \\
\hline Cavitation number & $0.5(\mathrm{~min})$ to $23(\mathrm{max})$ \\
\hline
\end{tabular}

Table 3. Propeller Axiom I characteristics

\begin{tabular}{l|l}
\hline Scale & $1: 1$ \\
\hline Number of blades & 3 \\
\hline Diameter $(\mathrm{m})$ & 0.3 \\
\hline Pitch / diameter ratio at $\mathrm{r} / \mathrm{R}=0.7$ & 0.847 \\
\hline Blade area ratio & 0.7 \\
\hline Direction of rotation & clock-wise \\
\hline
\end{tabular}

Table 4. Propeller Axiom II characteristics

\begin{tabular}{l|l}
\hline Scale & $1: 1$ \\
\hline Number of blades & 3 \\
\hline Diameter $(\mathrm{m})$ & 0.3 \\
\hline Pitch / diameter ratio at $\mathrm{r} / \mathrm{R}=0.7$ & 1.0 \\
\hline Blade area ratio & 0.5 \\
\hline Direction of rotation & clock-wise \\
\hline
\end{tabular}

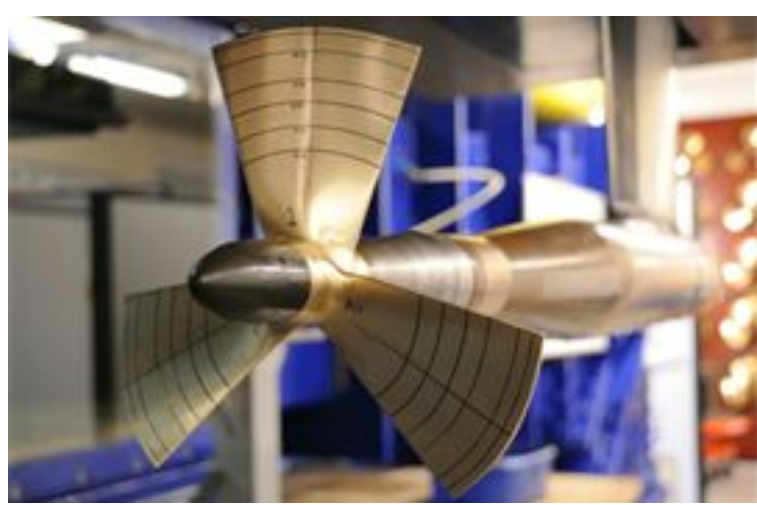

Fig. 7. The Axiom propeller prior to testing. 
Experiment with Axiom Propeller in Cavitation Tunnel

In order to assess the efficiency performance of the propeller, open water tests at atmospheric condition were conducted. The tests were performed to cover a practical range of advance coefficient $(\mathrm{J})$ varying between $\mathrm{J}=0.30$ and $\mathrm{J}=0.75$ under normal atmospheric conditions. For the tests the tunnel water speeds were held at $3.0 \mathrm{~m} / \mathrm{s}$ and $4.0 \mathrm{~m} / \mathrm{s}$, whilst the rotational rate of the propeller was varied to cover the above range of $\mathrm{J}$ values. Finally the data was non-dimensionalised using standard ITTC (2002) test procedures. The equations used in the analysis for the advance coefficient $(\mathrm{J})$, thrust coefficient $\left(\mathrm{K}_{\mathrm{T}}\right)$, torque coefficient $\left(\mathrm{K}_{\mathrm{Q}}\right)$, and open water efficiency $\left(\mathrm{n}_{0}\right)$, are given in Equations 1-4. Finally the test parameters were set using ECT procedures. The air content was held between $25-35 \%$ and Reynolds number based on resultant velocity at $0.7 \mathrm{R}$ gave a value typically $\operatorname{Re} \geq 1 \times 10^{6}$ and the cavitation number based on the free stream flow velocity of the tunnel was selected for practical convenience as shown in Equation 5.

$$
\begin{aligned}
& \text { Advance coefficient } \quad J=\frac{V}{n D} \\
& \text { Thrust coefficient } \quad K_{T}=\frac{T}{\rho n^{2} D^{4}} \\
& \text { Torque coefficient } \quad K_{Q}=\frac{Q}{\rho n^{2} D^{5}} \\
& \text { Open water efficiency } \quad \eta_{o}=\frac{K_{T}}{K_{Q}} \times \frac{J}{2 \pi} \\
& \text { Cavitation number } \quad \sigma_{o}=\frac{p-e}{\frac{1}{2} \rho V^{2}}
\end{aligned}
$$

Where, $\mathrm{V}$ is the tunnel free stream water velocity $(\mathrm{m} / \mathrm{s}), \mathrm{n}$ is the rotational speed of the propeller (rps), $\mathrm{T}$ is the thrust of the propeller $(\mathrm{N}), \mathrm{Q}$ is the torque $(\mathrm{N}-\mathrm{m})$ of the propeller, D is the propeller diameter $(\mathrm{m}), \mathrm{p}$ is the absolute pressure at the propeller disk $(\mathrm{Pa})$; $\mathrm{e}$ is the vapourisation pressure $(\mathrm{Pa})$ and $\rho$ is the density of the water in tunnel $\left(\mathrm{kg} / \mathrm{m}^{3}\right)$.

In addition to the open water tests, which represent the first quadrant performance of the propeller, i.e. the propeller working with positive rotational speed and positive advance velocity, the additional 3 quadrants were required. The 4 quadrant data therefore represents any flow condition experienced by a propeller during manoeuvring. During these tests the propeller may be rotated in the ahead (clockwise) or astern (anti-clockwise) directions while the direction of the tunnel flow was kept in the same (ahead) direction. The physical orientation of the propeller could have been changed back to front to simulate the appropriate quadrant according to the notation described below. However given the symmetrical nature of the design, only the first two quadrants were actually required the latter two $\left(3^{\text {rd }} \& 4^{\text {th }}\right.$ quadrant) being generated from the forme rowing to the skew symmetric nature of the data. In the case of a fixed pitch propeller it is conventional to define the four quadrants based on an advance angle $(\beta)$ defined in Equation 6. Using this nomenclature the 4

\begin{tabular}{|c|c|}
\hline $\begin{array}{l}2^{\text {nd }} \text { quadrant: } \\
\text { (Stopping in ahead) }\end{array}$ & $\frac{1^{\text {st }} \text { quadrant: }}{\text { (Going ahead) }}$ \\
\hline $\begin{array}{l}\text { Advance speed - ahead } \\
\text { Rotational speed - astern } \\
\text { Adv. angle } 90<\beta \leq 180\end{array}$ & $\begin{array}{l}\text { Advance speed - ahead } \\
\text { Rotational speed - ahead } \\
\text { Adv. angle } 0 \leq \beta \leq 90\end{array}$ \\
\hline $3^{\text {rd }}$ quadrant: & $4^{\text {th }}$ quadrant: \\
\hline (Reversing) & (Stopping in astern) \\
\hline Advance speed - astern & Advance speed - astern \\
\hline Rotational speed - astern & Rotational speed - ahead \\
\hline Adv. angle $180<\beta \leq 270$ & Adv. angle $270<\beta \leq 360$ \\
\hline
\end{tabular}
quadrants can be easily identified and are given in Table 5 .

$$
\text { Advance angle } \quad \beta=\tan ^{-1}\left(\frac{V}{0.7 \pi n D}\right)
$$

Table 5. The 4 quadrant propeller performance data

It should be noted that when $\beta=0^{\circ}$ or $\beta=360^{\circ}$ then this defines the ahead bollard pull condition and when $\beta=180^{\circ}$ this corresponds to the astern bollard pull situation. For $\beta=90^{\circ}$ and $\beta=270^{\circ}$, these positions relate to the condition when the propeller is not rotating and is being dragged ahead or astern through the water respectively. The hydrodynamic pitch angle defines the angle of the incoming velocity with the propeller plane. The magnitude of the incoming velocity vector is defined in Equation 7.

$$
\text { Incoming velocity } \quad V_{r}=\sqrt{V^{2}+(0.7 \pi n D)^{2}}
$$

The thrust coefficient $\left(\mathrm{C}_{\mathrm{T}}\right)$ and torque coefficient $\left(\mathrm{C}_{\mathrm{Q}}\right)$ for this analysis are defined using the resultant velocity and are given in Equations 8 and 9. The quadrant definitions used with the $\beta, C_{T}$ and $C_{Q}$ nomenclature follow the hydrodynamic angle of attack of the propeller blade. The $\beta, C_{T}, C_{Q}$ nomenclature had more consistency with propeller physics than the older quadrant definition used with the $\mathrm{J}, \mathrm{K}_{\mathrm{T}}$ and $\mathrm{K}_{\mathrm{Q}}$ nomenclature. 
Kwang-Cheol Seo

$$
\begin{aligned}
& \text { Thrust coefficient } C_{T}=\frac{T}{\frac{\pi}{8} \rho\left[V^{2}+(0.7 \pi n D)^{2}\right] D^{2}} \\
& \text { Torque coefficient } C_{Q}=\frac{Q}{\frac{\pi}{8} \rho\left[V^{2}+(0.7 \pi n D)^{2}\right] D^{2}}
\end{aligned}
$$

\section{Results and discussion}

The Axiom II propeller showed a marked improvement in terms of performance and cavitation over the previous design (Axiom I). To understand this increase the open water performance, 4 quadrant data and the cavitation patterns were analysed and compared to the Axiom I propeller.

\subsection{Open water analysis}

A plot of the open water performance of the Axiom II propeller is given in Fig. 8. The data is given for thrust $\left(\mathrm{K}_{\mathrm{T}}\right)$, torque $\left(10 \mathrm{~K}_{\mathrm{Q}}\right)$ and efficiency $\left(n_{0}\right)$ for all of the experimental points gathered. The data has been subsequently processed using least squares fit to give the backbone curves for each of these variables.

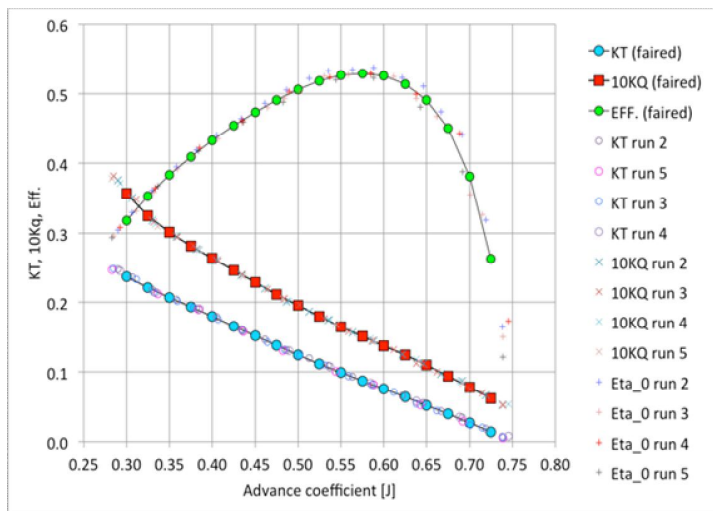

Fig. 8. Open water performance of the Axiom propeller II.

The Axiom II propeller provided very repeatable test data with only small test-to-test variation. The maximum efficiency was $53.7 \%$ at $\mathrm{J}=0.575$. When this was compared to the Axiom I propeller it is clear that the reduction in blade area and tapered blade outline as opposed to the plan form (square shape) between the two designs has had a significant positive effect on the efficiency. A comparison of the open water plots is given in Fig. 9 and it is clear that the new design was approximately $63 \%$ more efficient. The new propeller also has a wider range of achievable advance coefficients due to the increase in pitch ratio from $\mathrm{P} / \mathrm{D}=$ 0.86 to $\mathrm{P} / \mathrm{D}=1.0$ changing the operating point for the propeller from $\mathrm{J}=0.35$ (cavitating) to $\mathrm{J}=0.575$ (non-cavitating). This helped the Axiom II propeller operate in a virtually cavitation free condition at the design point.

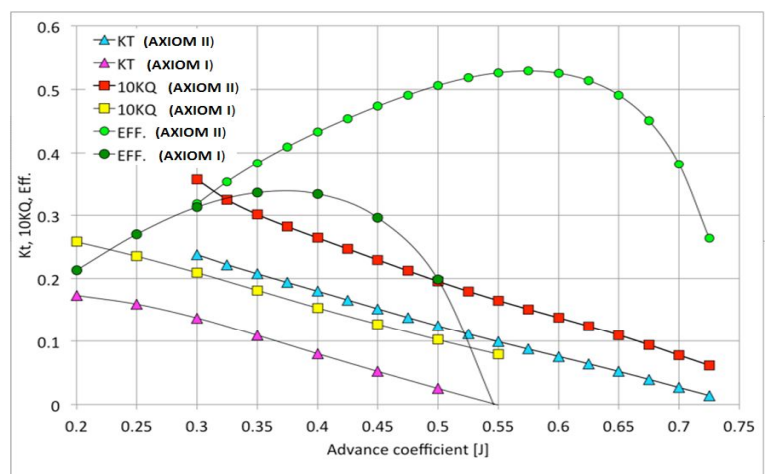

Fig. 9. Comparison of open water plots for the Axiom I and the Axiom $\Pi$ propeller.

\subsection{Multi-quadrant tests}

The Multi-quadrant tests were conducted by appropriately varying the tunnel flow speed (V), propeller shaft speed (n), direction of shaft speed (clockwise and anti-clockwise) and direction of tunnel flow (ahead and astern) via the relative position of the propeller with respect to flow as outlined in Section 2. Fig. 10 shows the results of the Axiom I propeller presented in the classical four quadrant notation of $\mathrm{C}_{\mathrm{T}}$ and $10 \mathrm{C}_{\mathrm{Q}}$ against $\beta$.

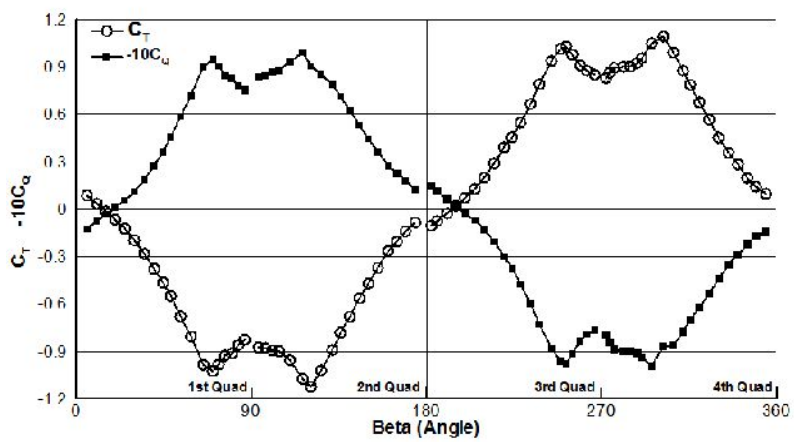

Fig. 10. Four quadrant data for the Axiom I propeller.

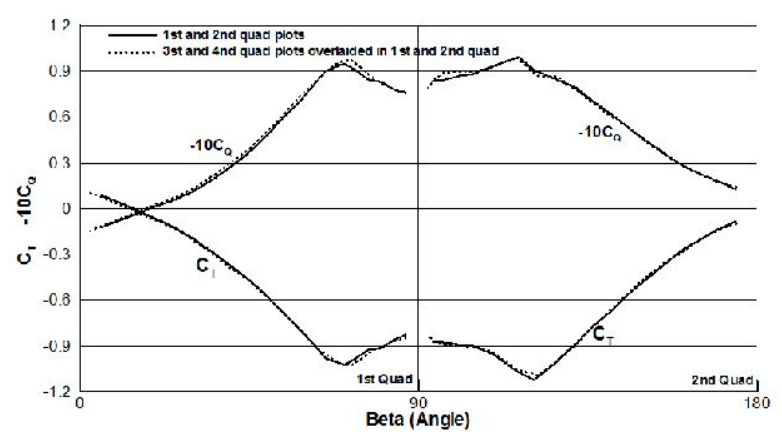

Fig. 11. Multi-quadrant data of the Axiom I propeller demonstrating the skew symmetry; the 3 rd and 4 th plots $\left(180^{\circ}-360^{\circ}\right)$ have been modified and overlaid into the 1st and 2nd quadrants. 


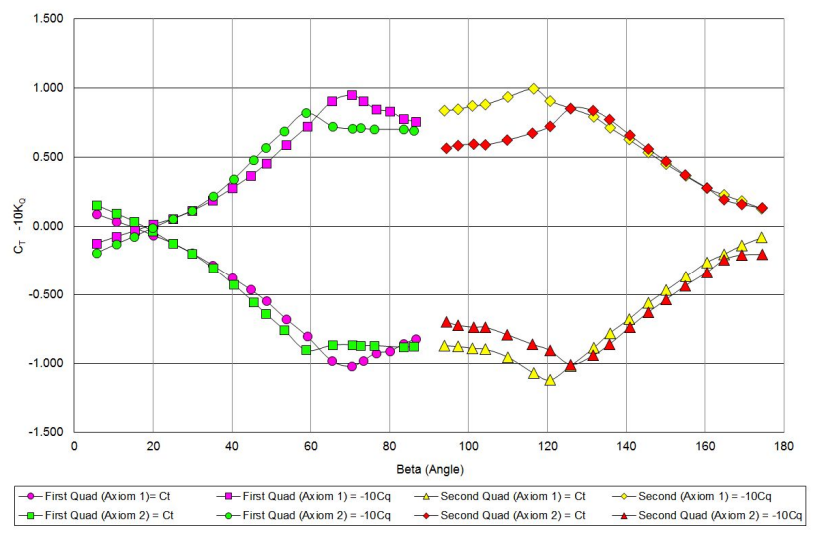

Fig. 12. Comparison of the Axiom I and Axiom II multiquadrant propeller data.

The Axiom propeller has skew symmetric characteristics in 1s tand 3rd quadrants (going ahead and astern) as well as 2nd and 4th quadrants (stopping in a head and astern).If minus is multiplied with $\mathrm{C}_{\mathrm{T}}$ and $10 \mathrm{C}_{\mathrm{Q}}$ of $3 \mathrm{rd}$ and 4 th quadrants and these curves are shifted to 1 st and 2 nd quadrants region the skew symmetry of $\mathrm{C}_{\mathrm{T}}$ and $10 \mathrm{C}_{\mathrm{Q}}$ curves can be found as replotted in Fig. 11; this is not the case for the conventional propellers, which are usually optimised for the forward motion only. Obviously this is a favourable attribute for the Axiom propeller for stopping and reversing as well as controlling the course keeping in both directions, ahead and astern, with almost similar performance. The small discontinuities around $\beta=0^{\circ}, 90^{\circ}, 180^{\circ}$, $270^{\circ}$ and $360^{\circ}$ are due to the physical limitations of the facility. These values can be obtained from the values around their vicinity by simple interpolation. In addition, in Fig.11 the Axiom I propeller data has been shown to be skew symmetric, which implied for this particular propeller that only the first two quadrants needed to be tested with the remaining data obtainable by simple arithmetic manipulation. When the results from the Axiom II propeller are overlaid on the plot, as shown in Fig. 12, it is clear that the Axiom II propeller performs slightly differently with the majority of the curve outside the Axiom I curve and the remaining $60^{\circ}$ of each quadrant inside.

\subsection{Cavitation observations}

The cavitation patterns on the Axiom II were similar to the first version of the propeller. The ' $\mathrm{S}$ ' type section is not ideally suited to heavily loaded conditions as it promotes significant levels cavitation mid chord on the blade. The cavitation inception began at approximately $\mathrm{J}=0.55$. At $\mathrm{J}=0.45$ the tip vortex

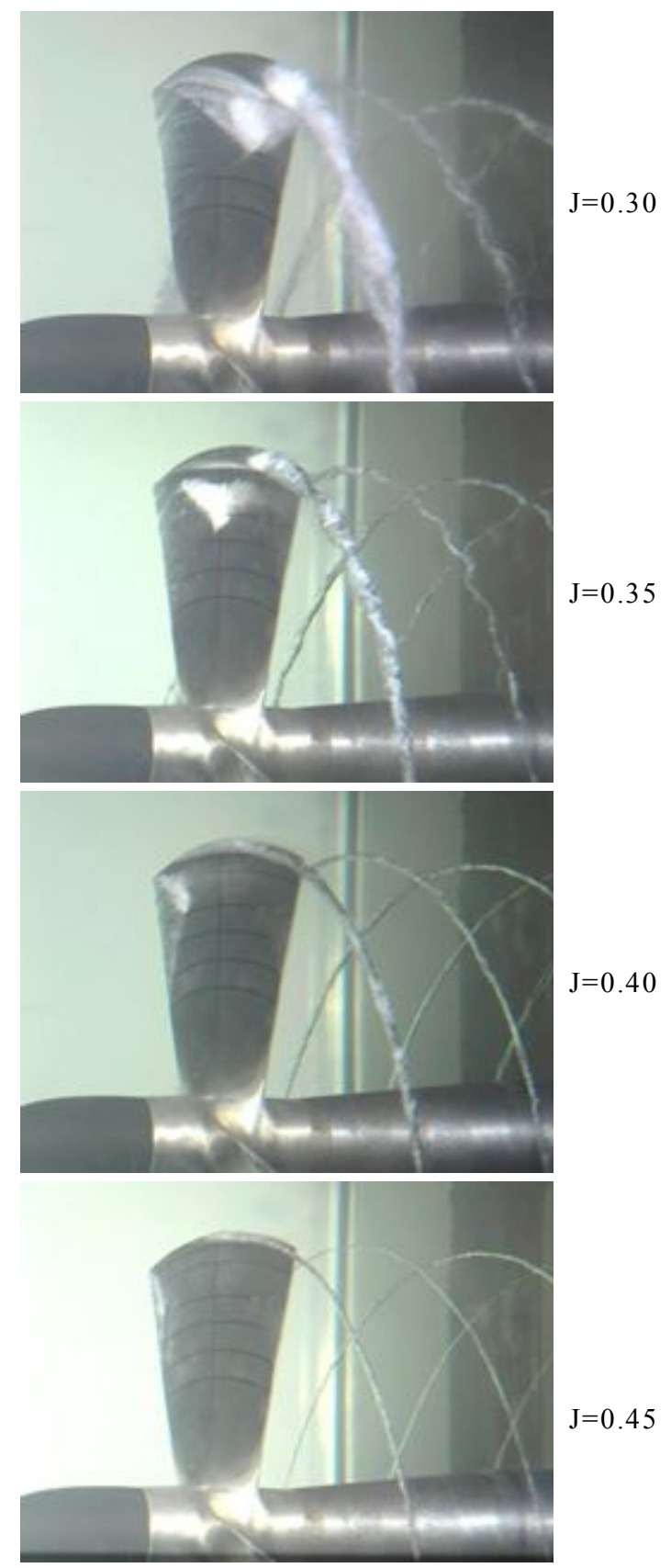

Fig. 13. Open water images $(\mathrm{J}=0.30-\mathrm{J}=0.45)$.

cavitation was a thin fully developed filament. At the leading edge between $\mathrm{r} / \mathrm{R}=0.7-0.9$ a small area of sheet cavitation began to develop. This sheet cavity would transit the chord as the $\mathrm{J}$ value was reduced to eventually combine with the tip vortex cavitation. However in this condition it extended approximately $5 \%$ of the chord. At $\mathrm{J}=0.40$ the cavitation types present on the blade began to stabilize. The sheet cavitation covered $10 \%$ of the blade mostly focused around $r / R=0.8$. However at this 


\section{Kwang-Cheol Seo}

condition the end of the sheet cavity was becoming unsteady and small wisps of erosive cloud cavitation could be detected. The tip vortex remained in position but increased in strength. By $\mathrm{J}=$ 0.35 the sheet cavitation covered half of the chord for each blade. The unsteady nature of the after part of the cavity was generating significant levels of mist cavitation, which would most certainly be erosive. The sheet cavity was also influencing the tip vortex cavitation, which too was starting to become unsteady and break down. At $\mathrm{J}=0.30$, the sheet cavitation covers more than $90 \%$ of the blade at $\mathrm{r} / \mathrm{R}=0.8$ and has begun to interact with the tip vortex cavitation. For this condition both the sheet and tip vortex cavitation are starting to break down and generate unsteady cavitation coupled with large amounts of erosive cloud cavitation. Cavitation observations were made with the propeller in the first and second quadrant runs at atmospheric condition. The results are given in Fig. 13 for a range of $J$ values $(J=0.30$ - $\mathrm{J}=0.45)$. For the tests, the tunnel was open to atmosphere; the flow velocity was kept constant $\left(\sigma_{o}=23\right)$, whilst the shaft rpm was varied to cover the range of operational conditions.
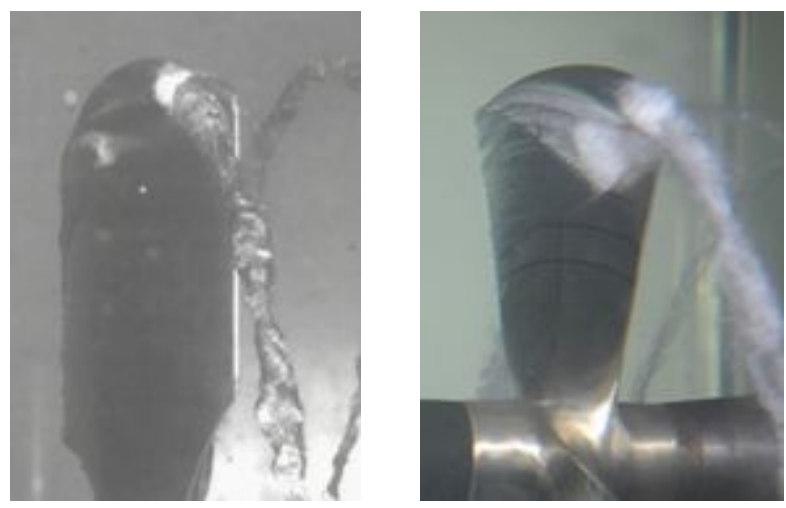

Fig. 14. Comparison of Axiom I (left) and Axiom $\Pi$ (Right) for $\mathrm{J}=0.30$ and $\mathrm{V}=3.0 \mathrm{~m} / \mathrm{s}$.

Fig. 14 shows a comparison of the Axiom I and the Axiom II propellers from the different tests. From this figure it is clear that the Axiom II is more heavily loaded at the same test condition, whereas the Axiom I with its smaller pitch ratio is still transitioning into unsteady cavitation range. The Axiom II , however, will typically operate cavitation free at $\mathrm{J}=0.55$ the tentative design point. Finally Fig. 15 shows the cavitation patterns for the multi-quadrant tests. In this figure it is easy to see the conventional cavitation pattern associated with first quadrant testing at $\beta=5^{\circ}$ however as the quadrant changes to the second quadrant by $\beta=175^{\circ}$, where the flow is forward and the propeller reversing the cavitation switched to the face of the propeller (pressure side). This condition would represent a transitory phase in stopping a vessel and not a steady state condition.

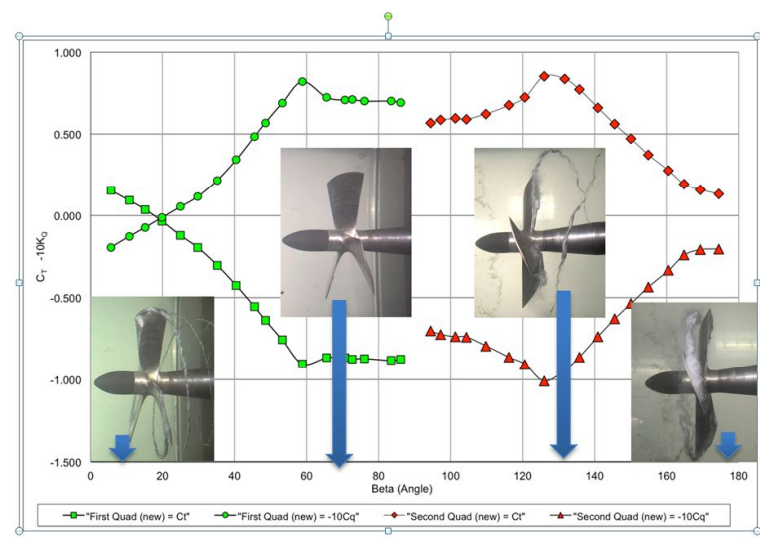

Fig. 15. Cavitation images from the multi quadrant tests.

\section{Conclusions}

This paper presented the cavitation tunnel tests for a $300 \mathrm{~mm}$ diameter, 3 bladed bi-directional thrust propeller. These tests were conducted to verify the propeller's efficiency the multi-quadrant performance and cavitation characteristics. Based on the tests it was found that:

1) Maximum efficiency of the Axiom II propeller was measured at $53 \%$; this was obtained during the first quadrant open water test.

2) Bearing in mind the differences in the $P / D, B A R$ and outline shapes, the Axiom II propeller can provide $63 \%$ more efficiency over the Axiom I propeller under similar conditions.

3) Useful, comparative multi-quadrant data for the two Axiom propellers are presented. The data reflected the symmetric feature of the propellers. The ahead and astern (thrust and torque) performance of this bi-directional thrust propeller was shown to have skew symmetry requiring only 2 quadrants to be tested.

4) In the first quadrant, the main cavitation patterns were a strong steady tip vortex and leading edge sheet cavitation at the suction (back) side of the blades. The extent and interaction of these cavities increased with reduced $\mathrm{J}$ value. The mid chord sheet cavitation was potentially erosive however the design point for this propeller is well away from the cavitation condition. 
Experiment with Axiom Propeller in Cavitation Tunnel

5) The Axiom II propeller with the new tapered outline shape would benefit from the inclusion of a duct to suppress cavitation and increase performance further. However the Axiom II design, for the condition shown, does not operate in a cavitation zone.

6) An overall powering evaluation of the Axiom propeller requires the validation of the delivered power which is a function of hull resistance and the propulsive efficiency. In order to do this evaluation either CFD analysis or model test experiment need to follow the overall evaluation of the Axiom propeller.

\section{References}

[1] Atlar, M.(2011), "Recent upgrading of marine testing facilities at Newcastle University", The second intl. conference on advanced model measurement technology for the EU maritime industry, Newcastle University, UK, 4-6 April.

[2] Chun, H. H., S. H. Kwon, D. D. Ha and S. U. Ha(1997), "An Experiment Study on the Course - Keeping of an 8,000 DWT Barge ship", Journal of the Society of Naval Architect of Korea, Vol. 34, No. 4, pp. 1-11.

[3] ITTC(2002), "Testing and Extrapolation Methods Resistance Test", International Towing Tank Conference, Technical report of the resistance committee, Procedure No. 7.5-02-02-01 3.6.2.

[4] Langley, M.(2009), "Tried \& tested - Axiom Propeller", Waterways World, WW Magazines UK, July, p. 94.

[5] Kim, S. Y., Y. G. Lee and S. H. Lee(2001), "A Study on the Flow Characteristics around a Hull Form of Coast-Canal Going Pusher-Barge", Journal of the Society of Naval Architect of Korea, Vol. 38, No. 1, pp. 9-26.

[6] Sampson, R., M. Atlar and G. Politis(2012), "AXIOM Mark II Propeller Multi - quadrant open water tests", Newcastle University MAST Technical Report MT/CT-2012-001.

[7] Seo, K. C., M. Atlar, J. Wightman-Smith and I. Paterson (2010), "Multi - quadrant open water tests with an Axiom propeller in the Emerson Cavitation Tunnel", Newcastle University MAST Technical Report MT/2010/00.

Received : 2014. 04. 10.

Revised : 2014. 05. 30.

Accepted : 2014. 06. 25. 